\title{
Intuitive Projection Display System Supporting the Manipulation of Dual Laser Pens
}

\author{
Ching-Sheng Wang, Ding-Jung Chiang, Chien-Liang Chen, and Hsin-Chueh Chen
}

\begin{abstract}
The combined use of computers and projectors in delivering multimedia presentations and in teaching is very common. However, the common operating tools used to deliver presentations such as presentation pens or mice either have limited manipulation functionality or are less convenient, and it is difficult to perform functions such as zooming, rotating images, and writing. In addition to allowing users to move freely in space to remotely perform functions, the laser pen manipulation system proposed in this paper can also perform the writing functions of a laser pen. Moreover, the system can also support the simultaneous use of two laser pens to realize a touch mode similar to that of a tablet computer, thus achieving an intuitive projection display manipulation experience.
\end{abstract}

Index Terms - Intuitive, laser pen, laser pointer, projection.

\section{INTRODUCTION}

The computer is a common piece of equipment in modern life, and the combined use of computers and projectors is widely applied in presentations and in teaching. However, the common computer presentation operating devices are mostly presentation pens and mice. Though people are very familiar with computer mice, they usually require the user to stay beside the computer and an operating table, thus resulting in restricted operation. In addition, writing is not smooth when using the mouse to perform writing functions. On the other hand, common and simple presentation pens have limited manipulation functions, such as laser guiding and page turning, and presentation pens with complex functions are often costly and unable to support the writing function. To this end, this paper proposed a set of intuitive projection display systems that are able to support the manipulation of two laser pens, thus enabling the most common laser pens to be used to perform a variety of manipulation and writing functions in an intuitive manner and deliver projected presentations efficiently.

With its light condensing and non-scattering characteristics, in addition to common guiding and presentation features, the laser pen has other various applications. For example, it can be used as the aiming point in a shooting game [1], [2] or to direct a robot arm to grab real

Manuscript received April 28, 2014; revised July 4, 2014. This work was supported in part by the Ministry of Science and Technology of the Republic of China, Taiwan under Grant NSC 102-2221-E-156 -010.

Ching-Sheng Wang, Chien-Liang Chen, and Hsin-Chueh Chen are with the Department of Computer Science and Information Engineering, Aletheia University, Taiwan (e-mail: cswang@mail.au.edu.tw, au1599@mail.au.edu.tw, fm020037@au.edu.tw).

Ding-Jung Chiang is with the Department of Digital Multimedia Design, Taipei Chengshih University of Science and Technology, Taiwan (e-mail: dingjung.chiang@gmail.com). objects [3]. The laser pen can also be combined with augmented reality to derive more multivariate interactive applications [4], [5]. In the proposed system, the laser pen is used to manipulate the computer cursor to enable the user to remotely control a computer without needing to stay beside it, thus increasing the opportunity to interact with others during teaching or conference presentations, and further enhancing the effect of the presentation.

Currently, many scholars are studying the use of laser pens or mobile devices to remotely control computers [6]-[10]. Pramit Dey et al. proposed a study on operating the window with a laser pen [6], however, was less intuitive compared the proposed system in this paper. Xiao-Jun Bi et al. designed a system called uPen [7] that can be combined with smart classrooms to enhance the interaction between teachers and students. However, because uPen is a specially designed hardware device, the cost is very high. Other scholars have used laser pens installed on smart-phones to facilitate the sharing of information and enhance interaction during presentations [8]. Gang Pan et al. used the gyroscope function in mobile devices to control a mouse cursor and manipulate a computer. However, the hardware costs of these methods are quite high [9]. The system proposed by Ching-Sheng Wang has good effects, but it has fewer functions and cannot support the manipulation of dual laser pens [10]. In summarizing the above-mentioned studies, the proposed system in this paper has more complete operating functions in addition to low building costs, and it can perform writing and double-click functions in addition to common functions such as page turning and click. The proposed system of this paper can also support dual laser pens to provide more intuitive manipulation functions such as zooming and rotation.

The remainder of this paper is organized as follows. Section II describes the system architecture and function, and Section III presents the system implementation tools and principle explanations, including light spot capture and coordinate calculation, as well as command judgment principles and implement methods. Section IV presents and explains the system results, and Section $\mathrm{V}$ provides a brief conclusion.

\section{SYSTEM ARCHITECTURE AND FUNCTION}

The manipulation scenario and system architecture of the intuitive laser pen proposed in this paper is shown in Fig. 1. The system can support three modes of operation, namely the 【Presentation Mode】 specially made for presentations, the 【Mouse Mode】 supporting the multiple mouse operating instructions, and the【Touch Mode】 for simulating the touch 
operation of mobile devices. The related functions supported in each mode are shown in Table I as follows:

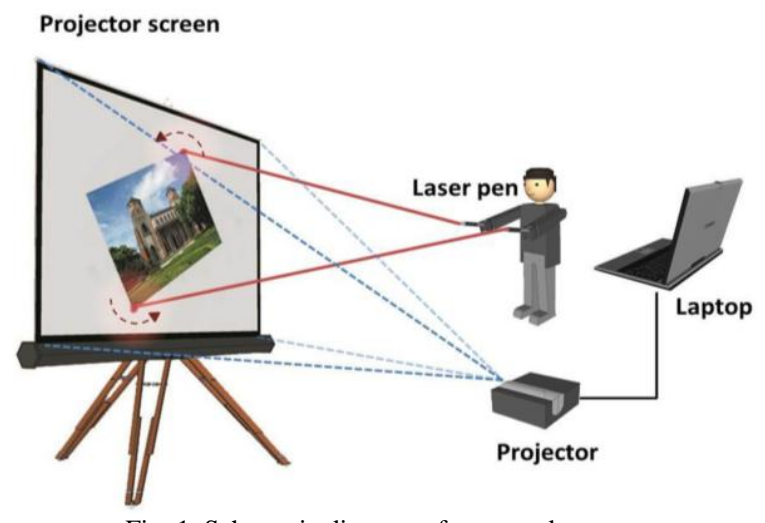

Fig. 1. Schematic diagram of proposed system.

TABLE I: TABLE OF SYSTEM FUNCTIONS

\begin{tabular}{cc}
\hline \hline Mode & Functions \\
Mode & Click, Page Turning, Write \\
\hline Mouse Mode & $\begin{array}{c}\text { Move, Click, Double-Click, Drag and Draw, } \\
\text { Zooming/Rotation(single laser pen), Right Button, } \\
\text { Middle Button }\end{array}$ \\
\hline Touch Mode & $\begin{array}{c}\text { Move, Click, Double-Click, Drag and Draw, } \\
\text { Zooming(dual laser pens), Rotation(dual laser pens) }\end{array}$ \\
\hline \hline
\end{tabular}

The【Presentation Mode】 has relatively simple functions and is mainly designed to provide the presenter with simple and convenient operating functions for delivering projected presentations. The functions provided in the 【Presentation Mode】 are primarily designed for MS PowerPoint, including [Click], [ Page Turning ], [Write ] and other functions. The [Click] function mainly corresponds to a mouse's click functions, such as switching to black or white screens and ending the presentation. During a presentation, page switching can be achieved by using the 【Page Turning】 function directly, and when the presenter wants to mark or add annotations to specific content it can be switched to the

[Write] function to perform the desired marking and writing actions.

The【Mouse Mode】supports multiple mouse operations, including [Move $],[$ Click $],[$ Double Click $],[$ Drag and Draw ], [Middle Button], 〔Right Button ], 〔 Zooming/Rotation ]. When applying the 〔Move function, the cursor will move along with the movement of the laser pen's light spot quickly to the location point of the light spot. The [Click ] function mainly simulates the action of clicking the left mouse button. The [ Double Click] function simulates the action of double click the left mouse button to perform the actions of opening a file or clicking for implementation. The

[Drag and Draw ] function simulates the object selecting and dragging function. The [Middle Button] function is mainly used to scroll up and down in the case of browsing a graphic simulation. The [Right Button ] function simulates clicking the right mouse button to open and select function options (e.g. copy or paste, etc.). The [Zooming/Rotation ] functions are mainly for simulating the zoom-in, zoom-out and simple rotation actions specific to pictures.

The【Touch Mode】mainly simulates the touch operation mode of a notebook or tablet computer's touch panel, and the [Move ], [Click], [Double Click] and [Drag and Draw ] functions are similar to the functions in the 【Mouse Mode 】. In addition, the [Zooming and [Rotation ] functions can be used with dual laser pens to further simulate the Zooming ] and [Rotation] actions on a touch panel. The proposed system is designed with the [Zooming] and [ Rotation ] functions specific to the simulated operations using either a single laser pen or dual laser pens. However, the use of dual laser pens is more intuitive because the judgment needs to be made in cooperation with the instructions in the case of a single laser pen, requiring the user to remember the operation instructions to complete the functional simulation. The use of dual laser pens is more similar to operating a touch panel, and thus is more intuitive and efficient compared with using a single laser pen.

\section{SySTEM IMPLEMENTATION}

The processing flowchart of the system is shown in Fig. 2, wherein the projected graphic is captured at the beginning in a real-time manner via the camera. The graphic is then converted into black and white through image binarization processing technology, and then the initial coordinates of the laser pen spot (white) is captured and the coordinate calculations and corrections are conducted. Finally the instruction analysis and output are performed through analyzing the on and off situations of the laser pen spot to further complete the simulation and performance of a variety of operating instructions.

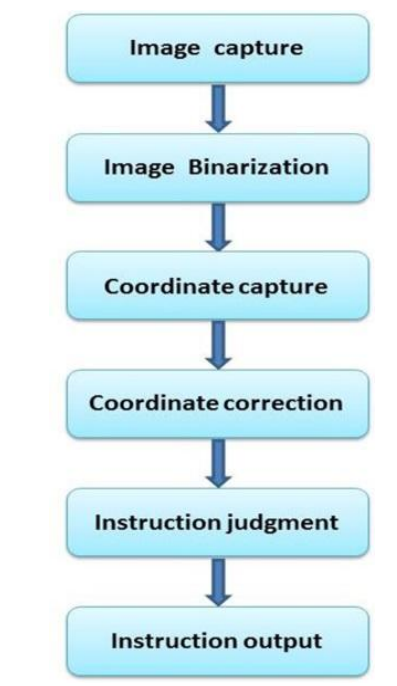

Fig. 2. System processing flowchart.

\section{A. Coordinate Calculation}

The system mainly uses C\# and the Emgu CV image processing library to implement the video graphic capture and processing. After the video information is captured by the camera, gray-level processing is first performed and then the appropriate thresholds are taken for binarization, through which the light spot of the laser pen (white) and the background (black) can be clearly distinguished. The coordinate calculation and correction of the light spot are then conducted. 
In addition, the system adopts a contour judgment method to calculate the initial coordinates of the light spot. First, the functions of Emgu CV FindContours are used to capture the contour and coordinates of the light spot. The central points of the four vertices in the contour at the top, bottom, left and right are then calculated as the initial coordinates of the light spot and the coordinates are corrected.

In the judgment of the coordinates of the dual laser pen, since two laser spots need to be tracked and analyzed at the same time, a multi-object judgment function is required. Specific to the multi-object judgment, and to enhance the system efficiency and functionality, the region judgment method was adopted in this paper. In this method, a graphic is divided into either the left and right regions or the upper and lower regions before performing the light spot contour judgment in individual regions, thus allowing the initial coordinates of the light spots of two laser pens to be captured in a fast and efficient manner.

When calculating the coordinates of the light spot, the graphic captured by the camera might be askew, and the resolution of the graphic captured by the camera could be different from that of the projector and computer; therefore, it needs to conduct resolution conversion and correction to match the resolution of the graphic captured by the camera to that of the projector, thus complete the light spot coordinate correction. The coordinate correction principle is shown in Fig. 3.

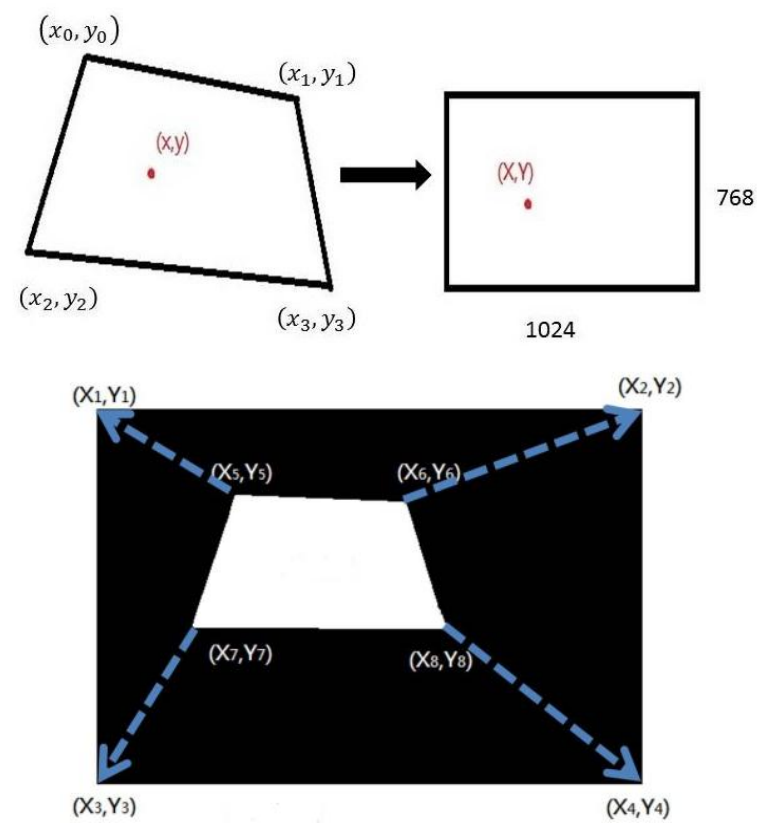

Fig. 3. Schematic diagram of coordinate correction.

$$
\begin{aligned}
& a=x_{1}-x_{0}+g x_{1} \quad g=\left|\begin{array}{cc}
\Sigma x & \Delta x_{2} \\
\Sigma y & \Delta y_{2}
\end{array}\right| /\left|\begin{array}{cc}
\Delta x_{1} & \Delta x_{2} \\
\Delta y_{1} & \Delta y_{2}
\end{array}\right| \\
& b=x_{3}-x_{0}+h x_{3} \quad h=\left|\begin{array}{cc}
\Delta x_{1} & \sum x \\
\Delta y_{1} & \sum y
\end{array}\right| /\left|\begin{array}{ll}
\Delta x_{1} & \Delta x_{2} \\
\Delta y_{1} & \Delta y_{2}
\end{array}\right| \\
& c=x_{0} \quad \Delta x_{1}=x_{1}+x_{2} \quad \Delta x_{2}=x_{3}-x_{2} \\
& d=y_{1}-y_{1}+g y_{1} \quad \Delta y_{1}=y_{1}+y_{2} \Delta y_{2}=y_{3}-y_{2} \\
& e=y_{3}-y_{0}+h y_{3} \quad \sum x=x_{0}-x_{1}+x_{2}-x_{3} \\
& y=y_{0} \quad \sum y=y_{0}-y_{1}+y_{2}-y_{3}
\end{aligned}
$$

The procedure is as follows: first select the coordinates of the four vertices of the graphic as the correction range; then assume the coordinates of the four vertices are $\left(x_{0}, y_{0}\right),\left(x_{1}\right.$, $\left.y_{1}\right),\left(x_{2}, y_{2}\right)$ and $\left(x_{3}, y_{3}\right)$ respectively and substitute them into (1) obtain the variables of $a, b, \ldots h$ as:

Substitute the obtained variables of $a, b, \ldots \mathrm{h}$ into the following equation for the coordinate conversion, where ( $x$, $y$ ) is the coordinate of the light spot captured by the camera and $(u, v)$ is the corrected coordinate obtained initially:

$(u, v)=\left(\frac{(e-f h) x+(c h-b) y+b f-c e}{(d h-e g) x+(b g-a h) y+a e-b d}, \frac{(f g-d) x+(a-c g) y+c d-a f}{(d h-e g) x+(b g-a h) y+a e-b d}\right)$

Substitute the obtained corrected coordinate $(u, v)$ into the resolution of the projector to obtain the final target coordinate. For example, in the case of the computer and projector resolution being $1024 \times 768$, the target coordinate $(X, Y)$ can be obtained by substituting the resolution into the following equation:

$$
(X, Y)=(1024 u, 768 v)
$$

\section{B. Judgment of Instructions}

In designing the simulated mouse operation, this paper referred to the previous method proposed by the author. The simulation was performed using the modified direct method [10], which enables the user to point at a target object accurately from a distance and implement the right instructions effectively. The proposed system is mainly divided into three operation modes, namely the 【Mouse Mode】,【Touch Mode】 and【Presentation Mode】. When the user wants to switch modes, he/she simply needs to move the mouse to the required mode without clicking it to achieve the function switch.

With the [Move] function, the cursor moves with the light spot of laser pen when the laser pen is turned on, and stops moving when the light spot disappears. In addition, functions such as [Click ], [ Double Click ], [Middle Button ] functions and [Right Button〕 functions remain in the mobile phase when the light spot is turned on, and the mouse can be moved freely to the intended objects and can implement the corresponding instructions when the light spot is turned off.

The [Drag and Draw ] function is divided into two parts, mainly, the move phase and drag phase. When the laser pen is turned on, it will first enter the move phase, in which the user can use the cursor to move the object and lock the object by leaving the cursor on it for about 0.3 seconds. Then in the drag phase, in which the drag action can be completed after the object is dragged to the target location and the laser pen is turned off.

The [Zooming/Rotation \ functions of a single laser pen are mainly based on the displacement, zooming scale and rotation direction of the light spot. In the case of performing actions within Windows Photo Viewer, using the laser pen to slide the light spot upward or downward can simulate the forward and backward sliding action of the mouse wheel to perform the zoom-in and zoom-out functions. In regards to the Rotation function, using the laser pen to slide the light 
spot to the right or to the left can perform a clockwise or counterclockwise rotation.

The [Zooming] and [Rotation] functions can be used with the dual laser pens to simulate the intuitive zooming and rotation functions of the【Touch Mode】. The distance variation of the two laser pens is used as the basis for intuitive [Zooming ] function. Sliding the light spots of the two laser pens outward opens the distance between them so as to perform the zooming-in function; conversely, the zooming-out function can be performed if the distance between the two light spots is decreased. As for the [ Rotation ] function, the clockwise or counter-clockwise rotation is determined by analyzing the relative displacement vector of the two light spots.

\section{DEMONSTRATION}

The camera of the system is not specifically restricted, and it can be the video camera of a laptop or an external webcam. There are few restrictions to the placement of the camera as long as it is able to capture the range of the projection screen. In the early stage of system implementation, in order to consider the brightness difference of the projection environment, the brightness and binarization value domain needs to be adjusted first so that the processed binarized graphic can present the light spot of laser pen clearly to enhance the stability of the system. After the binarization process is complete, the system projects the graphic via scanning, captures the four vertex coordinates of the projected graphic, and then covers the resolution to that of the projector through the coordinate correction, thus completing the automated four-corner positioning and coordinate correction.

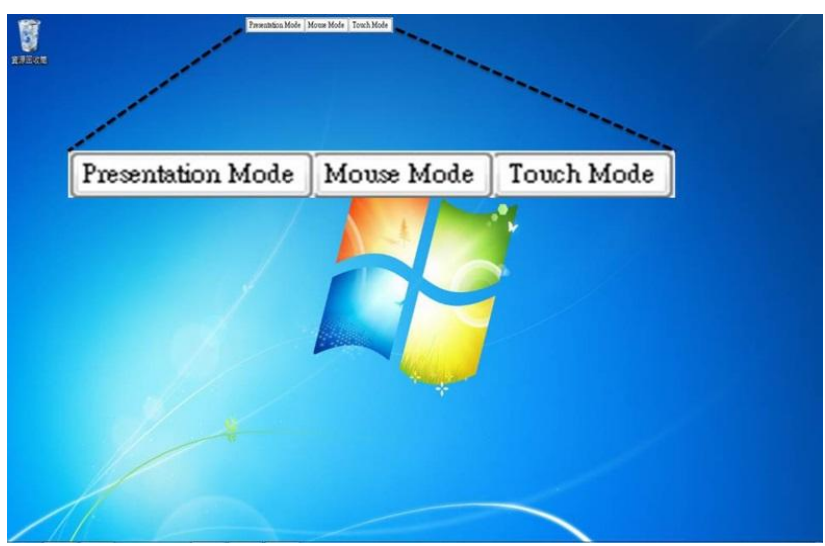

Fig. 4. Illustration of system function icons in main menu.

After the four-corner positioning and coordinate correction is complete, the main menu can be accessed to make free choices among the three kinds of operation modes (as shown in Fig. 4) and a variety of supporting functions of three operation modes (as shown in Fig. 5).

For example, if the【Mouse Mode】 in Fig. 4 is selected, it can switch to the function menu in the 【Mouse Mode】 as shown in Fig. 5(b) to further implement the instructions of Move ], [Click ], [Double Click ], 〔Drag and Draw ] , and other instructions. If the [Back ] function in Fig. 5(b) is selected, it can jump back to the main menu in Fig. 4.

In addition, the menu is placed separately at the top of the screen while the system is in operation and is set to always be displayed on top, to avoid it being blocked by other windows when the user wants to switch functions. When the user wants to switch between different functions, he/she only needs to move the mouse to the icon for that function, and the functions can then be switched smoothly.

\begin{tabular}{|l|l|l|}
\hline Click & Page Tuming \\
\hline
\end{tabular}

(a) Presentation mode

\begin{tabular}{|c|c|c|c|c|c|c|c|}
\hline Move & Click & Double Click & Drag and Draw & Zooming/Rotation & Right Button & Middle Button & Back \\
\hline
\end{tabular}

(b) Mouse mode

\begin{tabular}{|c|c|c|c|c|c|c|}
\hline Move & Click & Double Click & Drag and Draw & Zooming & Rotation & Back \\
\hline
\end{tabular}

Fig. 5. Illustration of function icons of three operation modes.

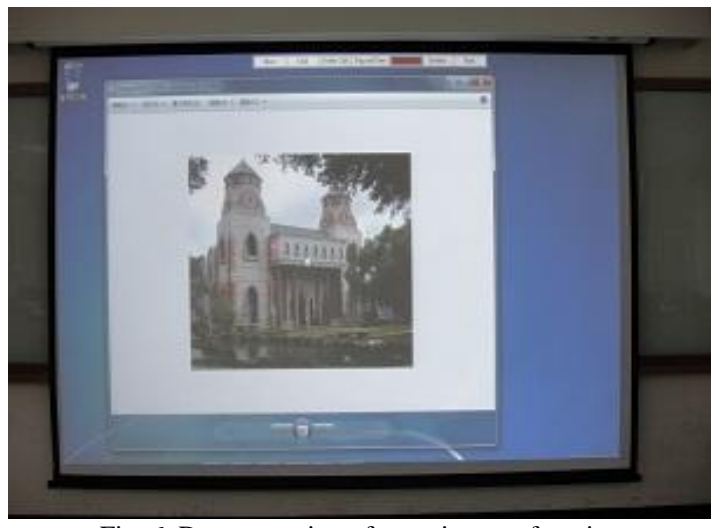

Fig. 6. Demonstration of zooming-out function.

Fig. 6 to Fig. 9 shows the results of system demonstration. Fig. 6 and Fig. 7 are the results after the zooming-in and zooming-out functions were performed, and Fig. 8) is the

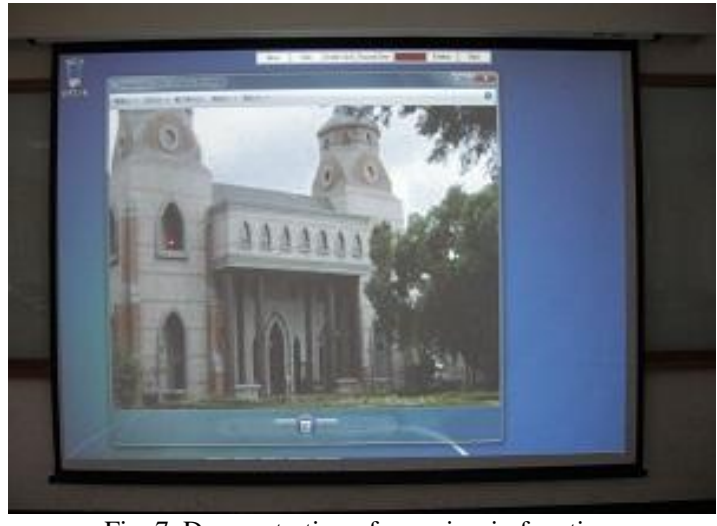

Fig. 7. Demonstration of zooming-in function.

result after performing a rotation function in MS Word. Fig. 9 is the result after performing the write function in MS PowerPoint, in which the content is marked and two English 
letters AU (abbreviation for Aletheia University) are written.

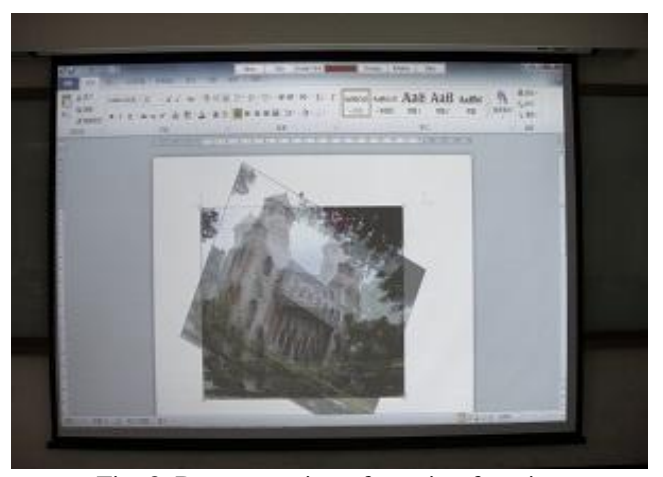

Fig. 8. Demonstration of rotation function.

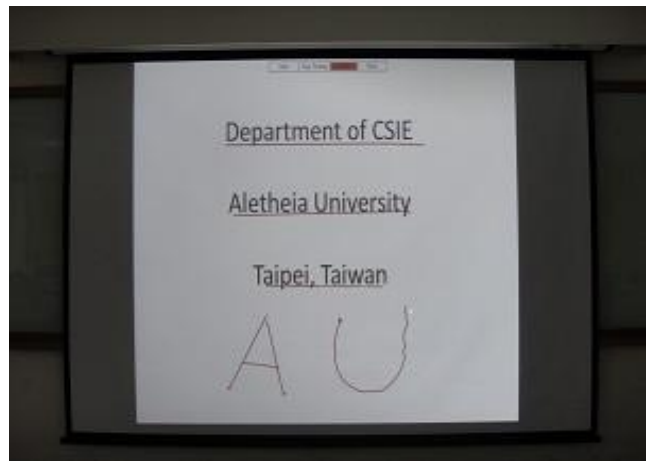

Fig. 9. Demonstration of write function.

\section{CONCLUSION}

In this paper, we proposed a projection display systems which able to support a set of intuitive manipulation function using either single or dual laser pens. The proposed system can support three effective operation modes based on the real-time image processing technology, the automated four-corner positioning and coordinate correction functions and the intuitive manipulation methods. Compared to other similar studies, the proposed method is not only more economical but also more intuitive and diverse in terms of its functions. In the future, we will try to develop the functions proposed in this paper on mobile device platforms such as iOS and Android, so that presenters can use their mobile devices instead of a personal computer and use the laser pen to operate the projection display system directly and efficiently.

\section{REFERENCES}

[1] W. K. Liou and S. C. Lee, "Application of laser guide and wireless control method on military training and the FPS game system," in Proc. 2012 IEEE 4th International Conference On Digital Game and Intelligent Toy Enhanced Learning, March 27-30, 2012, pp. 117-119.

[2] A. J. Junior, G. A. Gomes, N. A. Junior, A. D. Santos, C. A. Vidal, J. B. Cavalcante-Neto, and M. Gattass, "System model for shooting training based on interactive video, three-dimensional computer graphics and laser ray capture," in Proc. 2012 14th Symposium on Virtual and Augmented Reality, May 28-31, 2012, pp. 254-260.

[3] C. C. Kemp, C. D. Anderson, H. Nguyen, A. J. Trevor, and Z. Xu, "A point-and-click interface for the real world: Laser designation of objects for mobile manipulation," in Proc. the 3rd ACM/IEEE International Conference on Human-Robot Interaction, March 12-15, 2008, pp. 241-248.

[4] D. Kurz, F. Hantsch, M. GroBe, A. Schiewe, and O. Bimber, "Laser pointer tracking in projector-augmented architectural environments," in Proc. 6th IEEE and ACM International Symposium on Mixed and Augmented Reality, November 13-16, 2007, pp. 19-26.

[5] O. Oda and S. Feiner, "3D Referencing Techniques for Physical Objects in Shared Augmented Reality," in Proc. IEEE International Symposium on Mixed and Augmented Reality, November 5-8, 2012, pp. 207-215

[6] P. Dey, A. Paul, D. Saha, S. Mukherjee, and A. Nath, "Laser beam operated windows operation," in Proc. International Conference on Communication Systems and Network Technologies, May 11-13, 2012 , pp. 594-599.

[7] X. J. Bi, Y. C. Shi, and X. J. Chen, "uPen: A smart pen-liked device for facilitating interaction on large displays," in Proc. First IEEE International Workshop on Horizontal Interactive Human-Computer Systems, January 5-7, 2006, pp. 160-168.

[8] J. Seifert, A. Bayer, and E. Rukzio, "Pointer phone: Using mobile phones for direct pointing interactions with remote displays," in Proc. 14th IFIP Conference on Human-Computer Interaction, September 2-6, 2013, pp. 18-35.

[9] G. Pan, H. Y. Ren, W. D. Hua, Q. A. Zheng, and S. J. Li, "Easy Pointer: What you pointing at is what you get," in Proc. 29th ACM CHI Conference on Human Factors in Computing Systems, May 7-12, 2011, pp. 499-502.

[10] C. S. Wang, L. P. Hung, S. Y. Peng, and L. C. Cheng, "A laser point interaction system integrating mouse functions," World Academy of Science, Engineering and Technology, vol. 67, pp. 657-662, July 2010.

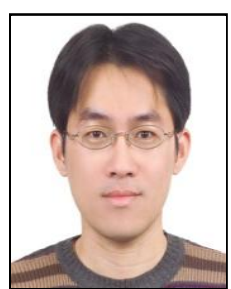

Ching-Sheng Wang received his $\mathrm{Ph}$. D. degree in the Department of Computer Science and Information Engineering from Tamkang University, Taiwan in 2001. Now, he is an associate professor and the chairman in the Department of Computer Science and Information Engineering at Aletheia University, Taiwan. His main research interests include indoor positioning, location-based service, ubiquitous computing, multimedia, virtual and augmented reality. He has published over 70 papers in international journals and conferences, and earned several research awards and grants, as well as participated in many international academic activities.

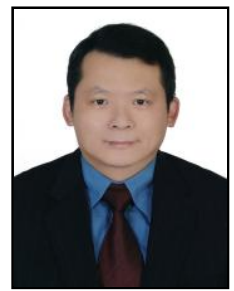

Ding-Jung Chiang received the Ph.D. degree in computer science and information engineering from Tamkang University in 2011 and received the bachelor and master degrees in computer science and information engineering from Tamkang University, Taipei, Taiwan, in 1995 and 1998, respectively. He is an assistant professor of the Department of Digital Multimedia Design at Taipei Chengshih University of Science and Technology, Taipei, Taiwan where he initially joined in August 1998. His research interests include ubiquitous computing, wireless communication and wireless multimedia. Dr. Chiang has published over 20 papers in international journals and conferences, and earned several research awards and grants, as well as participated in many international academic activities.

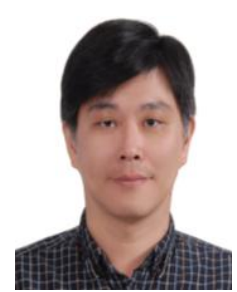

event systems.

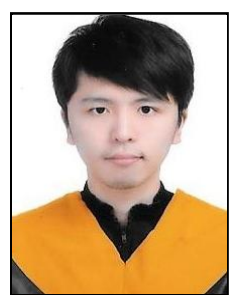

Chien-Liang Chen received the Ph.D. degree in electrical engineering from National Taiwan University in 2009. He was an assistant professor of tourism information at Aletheia University, where he initially joined in February 2010. Now, he is an assistant professor of computer science and information engineering at Aletheia University. His current research interests include design and analysis of algorithms, wireless sensor networks and discrete

Hsin-Chueh Chen is a master student in the Department of Computer Science and Information Engineering, Aletheia University, Taiwan. $\mathrm{He}$ received his B.S. degree in the Department of Computer Science and Information Engineering Aletheia University, Taiwan in 2013. 